\title{
Prevalencia, abundancia y caracterización de morfoespecies de sanguijuelas (Annelida: Hirudinea) en peces intermareales de Chile central ${ }^{\#}$
}

\author{
Prevalence, abundance and morphospecies characterization of leeches \\ (Annelida: Hirudinea) on intertidal fish from central Chile
}

\author{
P Moraga, G Muñoz*
}

Facultad de Ciencias del Mar y de Recursos Naturales, Universidad de Valparaíso, Valparaíso, Chile.

\begin{abstract}
SUMMARY
The aim of this study is to describe and compare the morphology and morphometry of leeches from intertidal fish. A total of 2,124 fish specimens that belonged to 15 species were collected from intertidal rocky pools from central Chile. They were externally examined for leeches. Twelve fish species harboured these parasites but only three of them had the sufficient number of individuals to perform morphometrical analyses. Five morphotypes of leeches were classified according to their colour and size; three morphotypes (MT1, MT2 and MT3) were from Scartichthys viridis, a morphotype (MT4) was from Sicyases sanguineus, and another morphotype (MT5) was from Aphos porosus. The five morphotypes were compared using six morphometrical measurements (length and width of the body, diameter of oral and posterior suckers, length of clitellum and position of clitellum from the anterior end). Morphometrical comparisons were based on absolute and relative measurements. The results indicated that MT4 and MT5 differed from the other morphotypes, whereas MT1, 2 and 3 did not differ significantly among them. MT4 had body and suckers wider than the other four morphotypes, whereas MT5 was longer, thinner and with a longer clitellum than the other morphotypes. According to these results, each fish species, of the three analyzed, harboured a distinct morphospecies. These leeches belong to the Piscicolidae family; although it was not possible to identify them at a genus level. This study also provides new records of leeches for nine intertidal fish species of Chile.
\end{abstract}

Palabras clave: parásitos, peces intermareales, morfometría, Chile. Key words: parasites, intertidal fish, morphometry, Chile.

\section{INTRODUCCIÓN}

Las sanguijuelas son organismos hematófagos que en la mayoría de los casos parasitan temporalmente a hospederos vertebrados que habitan en zonas terrestres o acuáticas, ya sean marinas o de agua dulce (Marshall y col 1980). Este grupo de parásitos ha sido escasamente estudiado en Chile y en general en toda Latinoamérica, por lo que existen pocos antecedentes taxonómicos y de registros en distintos hospederos y localidades (Muñoz y Olmos 2007). Esto último resulta importante en el avance de estudios parasitológicos ya que el reconocimiento de especies parásitas y de sus hospederos es crucial para estudios poblacionales y comunitarios incluyendo estudios de diversidad (Luque 2008) y de epidemiología (Rohde 1993).

Estos ectoparásitos pueden tener distintos grados de afecciones ya que al adherirse al hospedero mediante sus ventosas pueden dañar la superficie corporal de éstos, aunque usualmente no producen graves patologías (Burreson 1995).

\footnotetext{
Aceptado: 20.01.2010.

\# Proyecto FONDECYT 11060006.

* Casilla 5080, Reñaca, Viña del Mar, Chile; gabriela.munoz@cienciasdelmar.cl
}

Sin embargo, bajo ciertas circunstancias, tales como estrés ambiental, altas abundancias y tamaños corporales relativamente grandes (Burreson 1995), pueden afectar negativamente el crecimiento somático y la supervivencia de sus hospederos (Berven y Boltz 2001). Desde el punto de vista sanitario, las sanguijuelas pueden funcionar como vectores de microorganismos patógenos, tales como virus, bacterias y protozoos, a través de su modo de alimentación (Morillas y col 1987, Mulcahy y col 1990, Khan y col 2001, Kikuchi \& Fukatsu 2005, Khan y col 2008), provocando infecciones secundarias en las heridas que dejan después que se han alimentado (Burreson 1995).

Hirudinea contiene 12 familias, de las que dos han sido registradas en Chile: Glossiphonidae y Piscicolidae (Ringuelet 1985, Tello y col 2007). Según literatura, 10 especies de peces óseos cuentan con registros de sanguijuelas, de las cuales tres especies son del ambiente intermareal (Muñoz y Olmos 2007). De las especies determinadas están Platybdella chilensis en Aphos porosus (bagre de mar), Glyptonobdella sp. en Paralichthys sp. (lenguado) y Oceanobdella sp. en Bovichthys chilensis (torito); otros registros han sido indicados como "especies no identificadas de Piscicolidae" (Muñoz y Olmos 2007). Trabajos taxonómicos específicos de sanguijuelas parásitas de peces son los de Moore (1910), Ringuelet (1985), Szidat (1965) y Williams y col (2007). 
Este trabajo tiene como objetivos: 1) registrar e identificar las especies de sanguijuelas presentes en peces del ambiente intermareal mediante caracteres morfológicos y métricos; 2) determinar las abundancias y prevalencias de sanguijuelas en las especies de peces y su relación con el tamaño corporal de los hospederos.

\section{MATERIALES Y MÉTODOS}

Entre agosto de 2006 y diciembre 2007 se recolectaron 2.124 peces pertenecientes a 15 especies desde el inter-

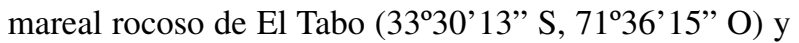

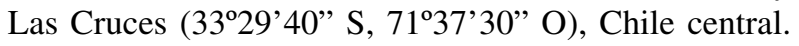
Los peces capturados fueron guardados individualmente en bolsas plásticas y congelados hasta el momento de su disección.

En el laboratorio, cada ejemplar fue revisado externamente para detectar y recolectar las sanguijuelas. Los parásitos fueron fijados en formalina al $10 \% \mathrm{y}$ algunos individuos fueron puestos previamente en agua salina caliente (entre $70-80^{\circ} \mathrm{C}$ ) para relajar su musculatura y conservar su forma normal. La magnitud del parasitismo se determinó a través de la prevalencia y de la abundancia promedio (Bush y col 1997) en cada especie de pez.

Distintos tamaños muestrales se obtuvieron para cada una de las quince especies de peces (cuadro 1); de ellas sólo tres tuvieron sanguijuelas en número suficiente para realizar descripciones morfológicas y análisis morfométricos:
Scartichthys viridis, Sicyases sanguineus y A. porosus. Las sanguijuelas fueron clasificadas según el patrón de pigmentación y morfología básica en cinco morfotipos (MT): tres morfotipos fueron obtenidos de S. viridis y designados como MT1 (sin pigmentación o líneas delgadas muy tenues), MT2 (completamente pigmentado) y MT3 (con líneas o bandas transversales), otro morfotipo de $S$. sanguineus, designado como MT4 (con pigmentación oscura y ventosas anchas), y un quinto morfotipo de A. porosus, designado como MT5 (más largo y delgado que los otros cuatro morfotipos) (figura 1). Las sanguijuelas de las otras especies de peces no fueron consideradas en los análisis estadísticos dado el bajo número de especímenes reunido. Sin embargo, igualmente fueron medidas y clasificadas según las características morfológicas y morfométricas para estos cinco morfotipos.

Las sanguijuelas fueron aclaradas en glicerina al $70 \%$ diluida en alcohol. Por medio de microscopios óptico y estereoscópico, se observaron algunas estructuras internas, tales como testículos, ovarios y el buche. Las mediciones corporales incluyeron seis caracteres: largo y ancho del cuerpo, diámetro de las ventosas anterior y posterior, largo del clitelo y distancia entre éste y el extremo anterior de la ventosa anterior. Las diferencias de cada medición corporal entre morfotipos fueron comparadas mediante un análisis de varianza (ANOVA) de una vía (Zar 1996). Al obtener diferencias significativas entre grupos, se aplicó la prueba $a$ posteriori de Schefeé para determinar qué grupos diferían del resto. Los datos fueron previamente transformados a

Cuadro 1. Especies y número de peces $(\mathrm{N})$ recolectados en la zona central de Chile, longitud total promedio (LT, cm) \pm desviación estándar (DE) de los peces, prevalencia $(\mathrm{P} \%)$ y abundancia promedio $(\mathrm{ABU}) \pm \mathrm{DE}$ de sanguijuelas durante el período comprendido entre agosto 2006 y diciembre 2007.

Species and numbers of fish collected $(\mathrm{N})$ in central Chile, average total length of fish $(\mathrm{LT}, \mathrm{cm}) \pm$ standard deviation $(\mathrm{DE})$, prevalence $(\mathrm{P} \%)$ and average abundance (ABU) $\pm \mathrm{DE}$ of leeches between August 2006 and December 2007.

\begin{tabular}{|c|c|c|c|c|c|}
\hline Especies & $\mathrm{N}$ & $\mathrm{LT} \pm \mathrm{DE}$ & $\mathrm{P} \%$ & $\mathrm{ABU} \pm \mathrm{DE}$ & Morfoespecie \\
\hline Auchenionchus microcirrhis & 63 & $9,5 \pm 5,9$ & 22,2 & $0,35 \pm 0,82$ & I \\
\hline Auchenionchus crinitus & 7 & $11,5 \pm 4,1$ & 28,6 & $0,28 \pm 0,48$ & I \\
\hline Auchenionchus variolosus & 5 & $16,1 \pm 3,1$ & 80,0 & $1,80 \pm 0,65$ & I \\
\hline Aphos porosus & 81 & $25,6 \pm 4,4$ & 12,3 & $0,14 \pm 0,13$ & III \\
\hline Bovichthys chilensis & 12 & $5,8 \pm 1,1$ & 16,6 & $0,16 \pm 0,38$ & I \\
\hline Helcogrammoides chilensis & 420 & $3,3 \pm 0,9$ & 1,2 & $0,02 \pm 0,01$ & I \\
\hline Helcogrammoides cuninghami & 8 & $3,1 \pm 0,2$ & 0 & 0 & \\
\hline Hypsoblenius sordidus & 49 & $4,6 \pm 1,4$ & 17,1 & $0,16 \pm 0,37$ & I \\
\hline Girella laevifrons & 257 & $10,0 \pm 5,5$ & 0 & 0 & \\
\hline Gobiesox marmoratus & 139 & $5,8 \pm 2,3$ & 0,7 & $0,01 \pm 0,08$ & I \\
\hline Graus nigra & 65 & $14,9 \pm 9,1$ & 0,7 & $0,03+0,25$ & I \\
\hline Myxodes cristatus & 2 & $4,2 \pm 1,3$ & 50,0 & $1,00 \pm-$ & no determinada \\
\hline Ophyogobius jenynsi & 3 & $6,7 \pm 0,9$ & 0 & 0 & \\
\hline Scartichthys viridis & 732 & $8,8 \pm 3,7$ & 36,7 & $1,08 \pm 2,60$ & I \\
\hline Sicyases sanguineus & 281 & $11,1 \pm 4,8$ & 3,2 & $0,10 \pm 0,82$ & II \\
\hline
\end{tabular}




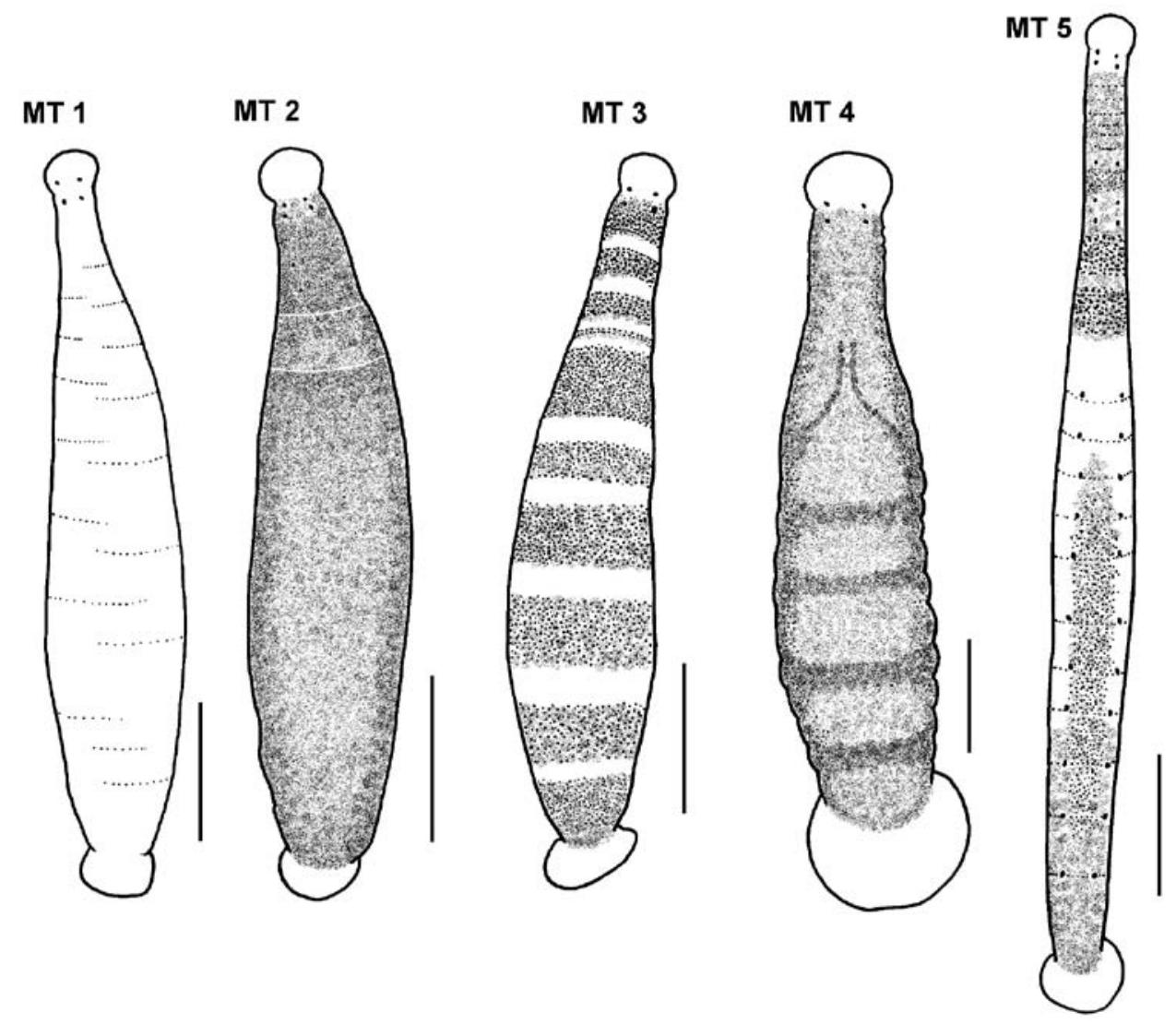

Figura 1. Morfología general y patrón de pigmentación de los cinco morfotipos (MT) de sanguijuelas encontrados en peces intermareales. Líneas de cota: $2 \mathrm{~mm}$.

General morphology and pigmentation of five leech morphotypes (MT) found on intertidal fish. Scale bars: $2 \mathrm{~mm}$.

$\log _{10}$ para ajustarlos a una distribución normal. También se aplicó el análisis de componentes principales (ACP) (Clarke y Warwick 1994), para realizar una ordenación espacial de los datos morfométricos en un gráfico de dos dimensiones y visualizar la formación de grupos entre los morfotipos de sanguijuelas.

Para identificar las sanguijuelas se realizaron preparaciones in toto, teñidas con hematoxilina, y se realizaron cortes histológicos longitudinales y transversales de cada morfotipo. Las claves utilizadas fueron las de Soós (1965), Knight-Jones (1962), Sawyer y col (1975), además de otras referencias específicas para algunas especies.

Para determinar si el tamaño corporal de los peces era un factor determinante en la parasitosis por sanguijuelas, se correlacionó la abundancia de estos parásitos con la longitud corporal de tres especies de peces con tamaños muestrales de parásitos y sanguijuelas suficientes para la correlación. Por otra parte, el promedio de abundancia y la prevalencia se correlacionó con la longitud total promedio de todas las especies de peces. Para todos estos casos se realizaron correlaciones de Spearman entre las variables mencionadas (Zar 1996). Todos los análisis estadísticos aplicados en este estudio fueron realizados en el programa Statistica, versión 8.

\section{RESULTADOS}

\section{COMPARACIÓN DE MORFOTIPOS}

Los cinco morfotipos mostraron variaciones morfométricas entre sí (figura 2), principalmente en la longitud corporal ( $\mathrm{P}<0,001)$, ancho corporal $(\mathrm{P}<0,001)$, ancho de las ventosas $(\mathrm{P}<0,001)$ y posición del clitelo $(\mathrm{P}<0,001)$. Los morfotipos con mayores variaciones fueron el MT4 y 5. El morfotipo MT4, de S. sanguineus, se diferenció de los otros cuatro morfotipos en todas las medidas corporales (a posteriori, $\mathrm{P}<0,05$ ), mientras que el MT5, de A. porosus, se diferenció de los otros principalmente en longitud corporal y del clitelo (a posteriori, $\mathrm{P}<0,05$ ) (figura 2 ). Sin embargo, los tres morfotipos de $S$. viridis (MT1, MT2 y MT3) no se diferenciaron morfométricamente entre sí, salvo en su coloración (figuras 1 y 2).

Las diferencias morfométricas entre morfotipos se observan gráficamente en el ACP tanto en los datos absolutos como en los relativos (figura 3). El FA1 (primer factor principal) explica en un gran porcentaje la varianza existente en los datos (70,35\%), mientras que el FA2 explica solamente un $14,52 \%$ de la varianza. Los datos absolutos presentan diferencias significativas entre los morfotipos 

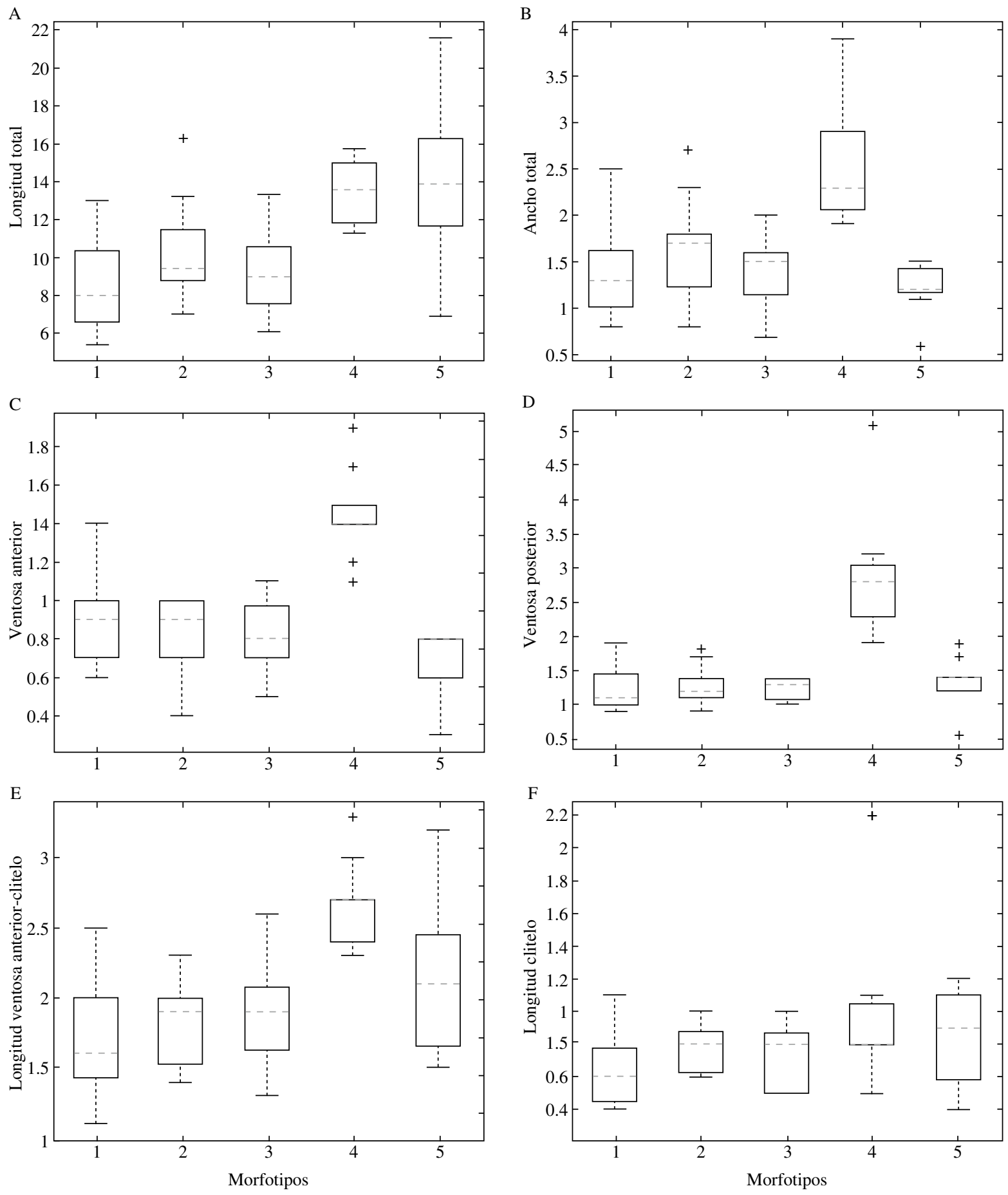

Figura 2. Promedios y variación morfológica de seis caracteres morfométricos, en medidas absolutas (mm), de los cinco morfotipos de sanguijuelas. La caja representa los datos desde el primer hasta el tercer cuartil, mientras que la línea punteada corresponde a la mediana, + son "outliers".

Averages and morphological variation of six morphometrical characters, in absolute values (mm), of five leech morphotypes. The box represents the data from the first to the third quartile, while the dotted line corresponds to the median, + are outliers.

(ANOVA para el eje 1 (FA1), $\mathrm{F}_{(4,64)}=18,9, \mathrm{P}<0,001$; eje 2 (FA2), $\left.\mathrm{F}_{(4,64)}=7,65, \mathrm{P}<0,001\right)$, distinguiéndose principalmente a MT4 y 5 , los cuales mostraron datos menos sobrepuestos entre sí y con respecto a los otros morfotipos. La longitud corporal de las sanguijuelas fue una de las variables que definieron el FA1, por lo tanto, fue necesario determinar si las diferencias observadas en los valores absolutos eran consistentes aun después 
de trabajar con los datos morfométricos relativizados a la longitud corporal. El porcentaje de variación bajó con respecto a lo observado en los datos absolutos (FR1 y FR2 explicaron una varianza significativa de los datos, 54,17 y 24,02, respectivamente), aunque se observó el mismo resultado anterior, es decir, MT4 y 5 son distintos, mientras que los MT1, 2 y 3 son similares entre sí (ANOVA eje 1 $(\mathrm{FR} 1){ }_{(4,64)}=31,65, \mathrm{P}<0,001$; eje 2 (FR2), $\mathrm{F}_{(4,64)}=3,09$, $\mathrm{P}<0,020$ ) (figura 3).

\section{DESCRIPCIÓN DE MORFOESPECIES}

Morfoespecie I: Morfotipos 1, 2 y 3 de Scartichthys viridis (agrupados de acuerdo a los resultados mostrados anteriormente). Descripción (45 ejemplares): Cuerpos alargados con pigmentación variable, sin pigmentación (blancas en general, algunas con tenues líneas delgadas que no otorgaban coloración), con líneas o bandas transversales pigmentadas y otras completamente pigmentadas (oscuras). Tenue metamerización. Cuerpo notoriamente distinguido entre traquelosoma y urosoma. Longitud corporal 5,4-16,3 mm. Ancho corporal 0,7-2,7 mm. Longitud del clitelo 0,4-1,1 mm a 1,1-2,6 mm, del extremo de la ventosa oral. Ventosas bien distinguidas, sin pigmentación ni ornamentación. Ventosa posterior 1,3 a 2,3 veces más ancha que la anterior. El diámetro de ambas ventosas no supera el ancho máximo del cuerpo. Dos pares de ocelos en el traquelosoma, cerca de la ventosa oral. Un par de ovarios tubulares pretesticulares. Cinco pares de testículos. No se observaron vesículas pulsátiles en el tegumento. Sitio de infección: superficie corporal, principalmente en las aletas pectorales. Depósito de material: Museo Nacional de Historia Natural de Chile, MNHN-HIRU $\mathrm{N}^{\text {os }} 1000,1001$ y 1002.

Morfoespecie II: Morfotipo 4 de Sicyases sanguineus. Descripción (15 ejemplares). Cuerpos alargados y pigmentados, la mayoría de coloración gris o café hacia la región anterior y más amarillenta hacia la posterior. La zona del clitelo menos pigmentada que el resto del cuerpo. Metamerización extremadamente marcada y el traquelosoma bastante más ensanchado, por lo que no se distingue tan fácilmente del urosoma. Longitud corporal 11,0-16,0 mm. Ancho corporal 1,9-3,9 mm. Longitud del clitelo 0,5-2,2 $\mathrm{mm}$ a 2,3-3,3 $\mathrm{mm}$ desde el extremo anterior de la ventosa oral. Ventosas bien distinguidas, sin pigmentación ni ornamentación. La ventosa posterior 1,7-2,7 veces más ancha que la ventosa oral. Diámetro de la ventosa posterior igual o superior al ancho máximo del cuerpo. Dos pares de ocelos en el traquelosoma. Un par de ovarios tubulares pretesticulares. Cinco pares de testículos. No se observaron vesículas pulsátiles en el tegumento. Sitio de infección: principalmente en sector ventral del cuerpo, a veces en las aletas. Depósito de material: Museo Nacional de Historia Natural de Chile, MNHN-HIRU No 1003.

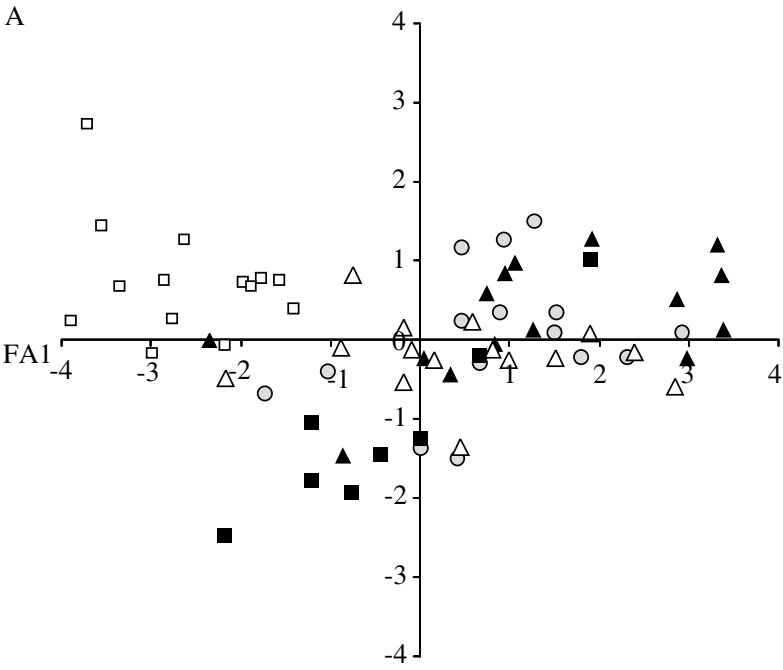

FA2
B

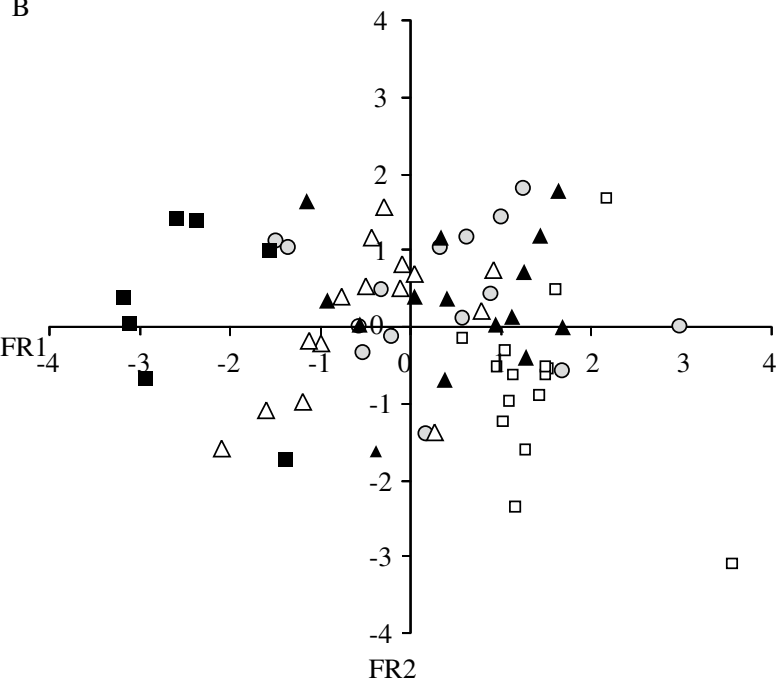

$\Delta$ MT1 $\triangle$ MT2 $\quad$ OMT3 $\quad$ वMT4 $\square$ MT5

Figura 3. Representación de los dos primeros factores composicionales del análisis de componentes principales (ACP) de las variables morfométricas para cinco morfotipos (MT) de sanguijuelas. A) Datos para los valores absolutos (FA) y B) para los valores relativos (FR).

Representation of two compositional factors from the principal components analyses (PCA) of morphometrical variables of five leech morphotypes (MT). A) Data for absolute values (FA) and B) for relative values (FR). 
Morfoespecie III: Morfotipo 5 de Aphos porosus. Descripción (9 ejemplares): Cuerpo alargado de tonalidad verdosa o café con bandas más oscuras a lo largo del cuerpo, especialmente en la parte central. El diámetro de ambas ventosas no supera el ancho máximo del cuerpo. Longitud corporal 6,9- 22,0 mm. Ancho corporal 0,6-1,5 mm. Longitud del clitelo 0,4-1,2 $\mathrm{mm}$ a 1,5-3,2 $\mathrm{mm}$ desde el extremo anterior de la ventosa oral. La región del clitelo se hace notar, presenta dos bandas más claras que lo delimitan. Ventosas bien distinguidas, sin pigmentación ni ornamentación. La ventosa posterior dos veces más ancha que la ventosa oral. Dos pares de ocelos en el traquelosoma y varios ocelos a lo largo del urosoma. Un par de ovarios pretesticulares, cinco pares de testículos y el cuerpo notoriamente distinguido entre traquelosoma y urosoma. No se observaron vesículas pulsátiles en el tegumento. Sitio de infección: sector ventral del cuerpo y branquias. Depósito de material: Museo Nacional de Historia Natural de Chile, MNHN-HIRU No 1004.

\section{REGISTROS DE SANGUIJUELAS Y TAMAÑO CORPORAL DEL} HOSPEDERO

La mayoría de las especies de peces recolectadas (12 de 15) presentaron sanguijuelas, aunque con abundancias y prevalencias variables (cuadro 1). La morfometría y morfología de las sanguijuelas encontradas en las tres especies de Auchenionchus, en $H$. chilensis, en $H$. sordidus y en $G$. nigra se asemejaban a la morfoespecie I, aunque con abundancias muy bajas en comparación a lo encontrado en Scartichthys viridis (cuadro 1).

La abundancia de sanguijuelas no se correlacionó con la longitud total de $S$. viridis $(\mathrm{N}=732, \mathrm{R}=0,48, \mathrm{P}>0,05)$, $S$. sanguineus $(\mathrm{N}=281, \mathrm{R}=0,06, \mathrm{P}>0,05) \mathrm{y} A$. porosus $(\mathrm{N}=81, \mathrm{R}=0,03, \mathrm{P}<0,03)$. A pesar de que las 15 especies de peces fueron de tamaños distintos, tampoco se encontraron resultados significativos al correlacionar la abundancia promedio y prevalencia de sanguijuelas con la longitud promedio de cada especie de pez $(\mathrm{n}=15$, $\mathrm{r}<0,03, \mathrm{P}>0,8$ para ambos descriptores).

\section{DISCUSIÓN}

En este estudio se encontró que doce de las quince especies de peces intermareales revisadas estaban parasitadas con sanguijuelas, agregándose nuevos registros de estos parásitos en nueve especies de peces de Chile. Esto indica que las sanguijuelas son parásitos comunes en peces intermareales y que la diversidad de éstos correspondería a por lo menos tres morfoespecies (I, II y III), distinguibles principalmente por su morfometría. La morfoespecie I, que incluye a tres morfotipos (MT1, 2 y 3), similares morfométricamente aunque de coloración variable, fue encontrada en $S$. viridis; la morfoespecie II contenía sólo al morfotipo 4 hallado en $S$. sanguineus, mientras que la morfoespecie III contenía sólo al morfotipo 5, encontrado en $A$. porosus (cuadro 1). Considerando las abundancias y prevalencias de las sanguijuelas en los distintos hospederos, se puede indicar que las morfoespecies II y III serían específicas para $S$. sanguineus y A. porosus, respectivamente, mientras que la morfoespecie I tendría una mayor variedad de hospederos; sin embargo, el hospedero principal sería $S$. viridis, ya que fue el que tuvo mayores abundancias y prevalencias de sanguijuelas.

Según este estudio, la longitud corporal de los peces no fue un factor relevante para la magnitud parasitaria de sanguijuelas, ya que no hubo ninguna correlación significativa entre prevalencia y abundancia de estos parásitos con respecto a la longitud de los peces. Es posible que la baja correlación entre estas variables sea consecuencia del carácter temporal que tienen estos parásitos sobre sus hospederos. De este modo, las magnitudes numéricas de sanguijuelas serían variables en hospederos de distinto tamaño, pero sin ninguna tendencia en particular.

En Chile se han registrado cinco familias de hirudíneos (Sielfeld 2002), de las cuales Piscicolidae y Glossiphonidae (Ringuelet 1985) contienes especies parásitas. Las tres especies de sanguijuelas encontradas pertenecen a Piscicolidae, caracterizada por ser ectoparásita de peces, presentar cuerpos cilíndricos y ventosa anterior notoriamente marcada del resto del cuerpo. A su vez, dentro de esta familia se encuentran seis géneros registrados para Chile, Branchellion Savigny, 1822, Platybdella Malm, 1863 (Sielfeld 2002), Austrobdella Badham, 1916 (Williams y col 2007), Oceanobdella Caballero, 1956 (Muñoz y col 2002), Glyptonobdella Sawyer y White, 1969 (Khan y col 2001) y Stibarobdella Schmarda, 1861 (Ringuelet 1981), que pueden ser posibles alternativas para las especies de sanguijuelas encontradas en los peces intermareales. Otro posible género es Malmiana Strand, 1942 ya que a pesar de que no cuenta con registros en nuestro país ha sido descrito en peces del intermareal, específicamente de la costa del norte de California en Estados Unidos (Burreson y Kalman 2006).

En este estudio se determinó que las tres morfoespecies no corresponden a los géneros registrados para Chile. Branchellion es descartado por presentar branquias externas en forma de hoja (Soós 1965), similar al género Stibarobdella, que además presenta tentáculos en su ventosa anterior (Soto 2000). Platybdella y Oceanobdella no cuentan con descripciones bien detalladas; sin embargo, Soós (1965) describió a Oceanobdella con ocelos dispuestos de forma circular en la ventosa posterior, tres pares de ojos, piel transparente y con un cuello cilíndrico distinguido del abdomen aplanado, mientras que Platybdella se caracteriza por tener dos o tres pares de ojos y con anillación no muy distinguida. Glyptonobdella se caracteriza por presentar cuerpos aplanados en la mayoría de sus especies, lo que es totalmente opuesto a lo observado en las morfoespecies estudiadas. En el caso de Malmiana, ninguna de sus características morfológicas, como ventosa oral relativamente larga con dos pares de ojos, uno de ellos en el 
traquelosoma y ocelos en la ventosa posterior (Burreson y Kalman 2006), fue observada en las morfoespecies del presente estudio.

La especie presente en $A$. porosus fue originalmente identificada como Platybdella chilensis (Moore 1910, Ringuelet 1985), aunque posteriormente Williams y col (2007) sugieren que correspondería a Austrobdella chilensis por presentar características morfológicas típicas de ese género, como ancho del cuerpo relativamente mayor a la ventosa posterior y por poseer un par de ojos en el traquelosoma, aunque no aportan más información al respecto. Sin embargo, Austrobdella se caracteriza por un canal celómico externo y vesículas pulsátiles en el tegumento, que no fueron observados en la superficie de los individuos ni en los cortes histológicos transversales realizados en este estudio.

Básicamente, ninguna de las morfoespecies de sanguijuelas encontradas en este estudio corresponde a los géneros de sanguijuelas posibles de encontrar en peces litorales de Chile. Es probable que se trate de nuevas especies e incluso de nuevos géneros de acuerdo al diagnóstico realizado por el Dr. Andrei Utevsky (Kharkov National University, Ucrania), quien se encuentra trabajando en la taxonomía de estos parásitos.

Finalmente, concluimos que las sanguijuelas son parásitos comunes de los peces intermareales de Chile central, reconociéndose tres morfoespecies, las que podrían ser nuevas para la ciencia, ya que según sus características morfológicas ninguna se asemejaba a los géneros registrados en la costa pacífica de Sudamérica y en peces intermareales de otros lugares. Este estudio además ha evidenciado que estos invertebrados son los menos conocidos dentro de la parasitofauna de Chile, lo que de por sí induce a continuar con estudios de cualquier aspecto biológico que contribuyan al conocimiento de estos parásitos.

\section{RESUMEN}

Este estudio describe y compara morfológica y métricamente a sanguijuelas de peces intermareales. Se recolectaron 2.124 peces de quince especies del intermareal rocoso de Chile central, los cuales fueron revisados externamente en búsqueda de sanguijuelas. Doce especies de peces albergaban a estos parásitos, pero sólo tres de ellas tenían números suficientes para compararlas morfométricamente. Se clasificaron cinco morfotipos de sanguijuelas según su color y tamaño; tres morfotipos (MT1, MT2 y MT3) eran de Scartichthys viridis, un morfotipo (MT4) de Sicyases sanguineus y de morfotipo (MT5) en Aphos porosus. Estos cinco morfotipos fueron comparados en seis medidas corporales (largo y ancho del cuerpo, diámetro de las ventosas anterior y posterior, largo del clitelo y distancia desde éste a la ventosa anterior). Las comparaciones morfométricas, tanto de los análisis basados en medidas absolutas y relativas, indicaron que MT4 y 5 eran distintos a los otros morfotipos, mientras que MT1, 2 y 3 no se diferenciaban significativamente entre sí. MT4 tenía un cuerpo robusto, con cuerpo y ventosas más anchas que los otros cuatro morfotipos y el MT5 era más largo, delgado y un clitelo más largo que el resto de los morfotipos. Según estos antecedentes, cada especie de pez, de las tres analizadas, albergaba una morfoespecie distinta. Estas sanguijuelas corresponden a la familia Piscicolidae, aunque no fue posible identificarlos a nivel de género. Este estudio además aporta con nuevos registros de sanguijuelas para nueve especies de peces intermareales de Chile.

\section{AGRADECIMIENTOS}

Se agradece el trabajo de la Sra. Edith Montero en la preparación de los cortes histológicos.

\section{REFERENCIAS}

Berven K, RS Boltz. 2001. Interactive effects of leech (Desserobdella picta) infection on wood frog (Rana sylvatica) tadpole fitness traits. Copeia 4, 907-915.

Burreson EM. 1995. Phylum Annelida: Hirudinea as vectors and disease agents. In: Woo PTK (ed). Fish Diseases and Disorders, Vol. 1: Protozoan and Metazoan Infections. CAB International, Oxford, UK, Pp 599-629.

Burreson EM, JE Kalman. 2006. A new species of Malmiana (Oligochaeta: Hirudinida: Piscicolidae) from tidepool fishes in northern California. J Parasitol 92, 89-92.

Bush AO, KD Lafferty, JM Lotz, AW Shostak. 1997. Parasitology meets ecology on its own terms: Margolis et al. revisited. J Parasitol 83, 575-583.

Clarke KR, RM Warwick. 1994. Change in Marine Communities: An Approach to Statistical Analysis and Interpretation. Plymouth Marine Laboratory, Plymouth, UK.

Díaz F, M George-Nascimento. 2002. Estabilidad temporal de las infracomunidades de parásitos de la borrachilla Scartichthys viridis (Valenciennes 1836) (Pisces: Blenniidae) en las costa central de Chile. Rev Chil Hist Nat 75, 641-649.

Khan RA, V Lobos, F Garcías, G Muñoz, V Valdebenito, M GeorgeNascimento. 2001 Cryptobia neghmei sp. n. (Protozoa: Kinetoplastida) en dos especies de lenguados Paralichthys spp. (Pisces: Paralichthydae) de la costa de Chile. Rev Chil Hist Nat 74, 763-767.

Khan RA, F Díaz, M George-Nascimento. 2008. Two new protist species, Trypanoplasma ojedae sp. n. (Mastigophora: Kinetoplastida) and Trichodina lascrucensis sp. n. (Ciliophora: Peritrichida) in a blennid fish, Scartichthys viridis, from the coast of Chile. Rev Biol Mar Oceanogr 43, 585-590.

Kikuchi Y, T Fukatsu. 2005. Rickettsia infection in natural leech population. Microb Ecol 49, 265-271.

Knight-Jones EW. 1962. The systematics of marine leeches. In: Mann $\mathrm{KH}$ (ed). Leeches (Hirudinea): their structure, physiology, ecology and embryology. Pergamon Press, Oxford, UK, Pp 169-186.

Luque JL. 2008. Parásitos: ¿Componentes ocultos de la Biodiversidad? Biologist (Lima) 6, 5-7.

Marshall AJ, WD Williams, FP Casanovas, TJ Parker. 1980. Zoología Invertebrados, Vol. 1. Editorial Reverté, Barcelona, España.

Moore JP. 1910. Platybdella chilensis sp. nov. Rev Chil Hist Nat 14, 28-30.

Morillas J, M George Nascimento, H Valeria, RA Khan. 1987. Trypanosoma humboldti $\mathrm{n}$. sp. from the Chilean catshark, Schroederichthys chilensis (Guichenot, 1848). J Protozool 34, 342-344.

Mulcahy D, D Klaybor, WN Batts. 1990. Isolation of infectious hematopoietic necrosis virus from a leech (Piscicola salmonisitica) and a copepod (Salmincola sp.), ectoparasites of sockeye salmon Onchorhynchus nerka. Dis Aquat Org 8, 29-34.

Muñoz G, V Valdebenito, M George-Nascimento. 2002. La dieta y la fauna de parásitos metazoos del torito Bovichthys chilensis Regan 1914 (Pisces: Bovichthydae) en la costa de Chile centro-sur: variaciones geográficas y ontogenéticas. Rev Chil Hist Nat 75, 661-671.

Muñoz G, V Olmos. 2007. Revisión bibliográfica de especies ectoparásitas y hospedadoras de sistemas acuáticos de Chile. Rev Biol Mar Oceanogr 42, 89-148.

Ringuelet RA. 1945. Hirudíneos del Museo de la Plata. Rev Mus La Plata 4, 95-137. 
Ringuelet RA. 1981. Los hirudíneos del Museo de Historia Natural de Montevideo. Com Zool Mus Hist Nat Montevideo 11, 1-39.

Ringuelet RA. 1985. Sinopsis de los hirudíneos de Chile (Annelida). Bol Soc Biol Concepción, Chile, 56, 163-179.

Rohde K. 1993. Ecology of Marine Parasites. $2^{\text {nd }}$ ed. CAB International, Oxon, UK

Sawyer R, AR Lawler, RM Oversrteet. 1975. Marine leeches of the eastern United States and the Gulf of Mexico with a key to the species. J Nat Hist 9, 633-667.

Sielfeld W. 2002. Annelida, Hirudinea. Guías de Identificación y Biodiversidad Fauna Chilena. Apuntes de Zoología, Universidad Arturo Prat, Iquique, Chile.

Soós A. 1965. Identification key to the leech (Hirudinoidea) genera of the world, with a catalogue of the species. I. Family: Piscicolidae. Acta Zool Acad Scient 11, 417-463.
Soto JMR. 2000. Marine leech, Stibarobdella macrothela (Schmarda, 1861) (Hirudinea: Piscicolidae), parasitic on the whaler shark, Carcharhinus brachyurus (Günther, 1870) (Chondrichthyes, Carcharhinidae), in Southern Brazilian waters. Rev Brasil Biol 60, 713-714.

Szidat L. 1965. Estudios sobre la fauna de parásitos de peces antárticos. I. Los parásitos de Notothenia neglecta Nybelin. Servicio de Hidrología Naval, Secretaría de Marina, Buenos Aires, Argentina.

Tello P, V Jerez, VL Olmos. 2007. Sanguijuelas (Hirudinea: Glossophoniidae) asociadas a Cryphios caementarius (Decapoda: Palaemonidae) del Río Limarí, Chile. Rev Biol Trop 55, 255-260.

Williams JI, PM Urrutia, EM Burreson. 2007. Two new species of Austrobdella (Hirudinea: Piscicolidae) from Chile. J Parasitol 93, 184-189.

Zar JH. 1996. Biostatistical Analysis. Prentice Hall International, New Jersey, USA. 